\title{
Simplified and objective assessment of spermatogenesis in spinal cord injured men by flow cytometry analysis
}

\author{
I H Hirsch MD,${ }^{1} \mathrm{D}$ Kulp-Hugues MD,${ }^{1} \mathrm{~J}$ Sedor $\mathrm{MS},{ }^{1} \mathrm{P}$ McCue $\mathrm{MD},{ }^{2} \mathrm{M}$ B Chancellor \\ MD,$^{1} \mathrm{~W}$ E Staas MD ${ }^{3}$ \\ Deparments of ${ }^{1}$ Urology, ${ }^{2}$ Pathology, ${ }^{3}$ Physical Medicine and Rehabilitation, Regional \\ Spinal Cord Injury Center of the Delaware Valley, Jefferson Medical College, \\ Philadelphia, PA, USA.
}

\begin{abstract}
Deterioration of the germinal epithelium of the testis is a known sequela of spinal cord injury (SCI) that may influence the outcome of male reproductive rehabilitation efforts. Quantitative testicular biopsy, currently regarded as the standard of assessing the integrity of spermatogenesis, has not gained widespread clinical use because of its invasive nature and relative technical complexity. Alternatively, aspiration DNA flow cytometry analysis of the testis has offered a potential method of spermatogenic assessment that meets both the requirements of simplicity and objectivity. The objective of this study is to determine the capability of flow cytometry to assess spermatogenesis following SCI. Eleven SCI men underwent incisional testicular biopsy with the specimen simultaneously submitted for quantitative evaluation of the germinal epithelium by both quantitative histometry and DNA flow cytometry. The haploid percentage of cells showed highly significant levels of correlation with key micrometric parameters of the quantitative testicular biopsy: spermatid/tubule $(p<0.002)$ and the spermatid/Sertoli cell ratio $(p<0.0005)$. Since tissue procurement is accomplished less invasively for flow cytometry analysis, we recommend this method as the modality of assuring integrity of the germinal epithelium in candidates for reproductive rehabilitation.
\end{abstract}

Keywords: spermatogenesis; spinal cord injury; flow cytometry analysis.

\section{Introduction}

The estimated prevalence of spinal cord injury approaches 200,000 in the USA with most victims being males in their reproductive years of life. ${ }^{1}$ Understandably, reproductive function ranks high among the priorities of global rehabilitation goals set forth by newly injured SCI patients. Therefore, the Task Force on Medical Rehabilitation Research recently established, as a general priority, the investigation of mechanisms that underlie infertility in SCI men and the development of strategies for restoring or improving fertility. ${ }^{2}$ Because of the resultant ejaculatory failure in up to $90 \%$ of those affected, spinal cord injured men have

Correspondence: Dr Hirsch, Department of Urology, Jefferson Medical College, 1025 Walnut St (Room 1112), Philadelphia, PA 19107, USA. historically been considered virtually sterile with fertility rates ranging from $0 \%$ to $5 \%$ in various reports. Therefore, the introduction of electroejaculation has been one of the major achievements in reproductive medicine during the past decade as successful semen recovery can now be accomplished in $80-90 \%$ of SCI men so treated. Among the urologic sequelae of SCI, neurogenic infertility has received increasing attention with the application of new semen recovery techniques and assisted reproductive technologies. Despite these methods, only $10 \%$ of SCI men entering reproductive rehabilitation programs are able to achieve pregnancy by intrauterine insemination ${ }^{3}$ and $30 \%$ by in vitro fertilization. ${ }^{4}$ In addition to enhancing male fertility potential following SCI, the availability of predictable semen recovery methodology has offered new 
avenues of clinical research in male reproductive rehabilitation. A review of these studies has shown that male reproductive dysfunction following SCI is of multifactorial origin with abnormalities demonstrable in the testis, the male conduction system, and the accessory sex glands. Of these factors, the one most widely studied has been the problem of spermatogenic dysfunction which has been reported in up to $90 \%$ of men following SCI. ${ }^{5}$ Therefore, failure of sufficient sperm production may limit fertility potential in candidates entering male reproductive rehabilitation programs. Objective assessment of spermatogenesis can most readily be achieved by the quantitative testicular biopsy; however, this procedure is both invasive and labor intensive. Alternatively, spermatogenic assessment by means of DNA flow cytometry analysis provides a potentially rapid and objective quantitative technique that has the advantage of tissue recovery by fine needle aspiration. While this method has shown good concordance with the qualitative testicular biopsy, ${ }^{6}$ it has not as yet been studied in conjuction with the quantitative testicular biopsy in spinal cord injured men. Therefore, the objective of this study is to determine the accuracy of flow cytometry as a technique for assessing spermatogenesis following SCI by comparison to the parameters of the quantitative testicular biopsy, the current standard of objective spermatogenesis assessment.

\section{Materials and methods}

Entering a program of reproductive rehabilitation were 11 spinal cord injured men (ages 22-43) with lesions ranging from C5 to T10. The mean duration of injury was 6.8 years and, in 8/11 men, the degree of SCI was complete. These patients underwent unilateral incisional testicular biopsy under either local or no anesthesia as part of a comprehensive assessment of fertility potential and suitability for stimulated semen recovery. The $2-3 \mathrm{~mm}$ specimen of testicular parenchyma was equally divided for parallel analysis by both standard quantitative micrometric techniques and DNA flow cytometry analysis.

\section{Quantitative testicular biopsy}

Quantitative analysis of the seminiferous tubules and epithelial parameters was performed on incisional testicular biopsies fixed in Bouin's solution. The tubular diameter and tubular wall thickness were measured by $\mathrm{AO}$ filar micrometer eye pieces and the number of Sertoli cells and late spermatids per seminiferous tubule were counted under $400 \times$ magnification. Using the Clermont's cytologic description of the human seminiferous epithelium, micrometric analysis was performed counting only germinal cell elements in the later stages of spermatogenesis $(\mathrm{Sc}+\mathrm{Sd}) .^{7}$ For each patient, at least 10 randomly selected round seminiferous tubules were selected for analysis. The mean concentration of Sertoli cells and mature spermatids per tubule was determined. The mean tubular wall thickness was measured from photomicrographs obtained at a magnification of $400 \times$ with $40 \%$ enlargement of the print. These parameters were measured with a photomicrograph of a micrometer at identical magnification and enlargement. The following quantitative histometric values were assessed for each patient: mean concentration of late spermatids per tubule (spermatid/tubule), mean ratio of spermatids Sertoli cells per tubule (spermatid/Sertoli cell), and tubular wall thickness.

\section{DNA flow cytometry analysis}

Testicular specimens were mechanically dissociated by forcefully injecting the tissue sample through a 22-gauge needle until the cells were completely dispersed in Hank's Balanced Salt Solution. ${ }^{a}$ The resulting cell suspension was then rinsed into a $50 \mathrm{ml}$ conical test tube and centrifuged at $400 \times \mathrm{g}$ for 10 minutes. Following removal of the supernatant, the cell pellet was resuspended in citrate buffer solution ${ }^{b}$ and adjusted to a concentration of $3 \times 10^{6}$ cells $/ \mathrm{ml}$. Polypropylene freezing vials ${ }^{\mathfrak{c}}$ containing aliquots of $0.2 \mathrm{ml}$ were frozen at $-80^{\circ} \mathrm{C}$. Within 14 days, an aliquot was thawed at room temperature, and the cell suspension transferred to fresh citrate buffer $(2 \mathrm{ml})$ and centrifuged at $400 \times \mathrm{g}$ for 10 minutes. All but $0.2 \mathrm{ml}$ of the supernatant was removed, 
the cell pellet was dispersed, and $1.8 \mathrm{ml}$ of detergent buffer containing trypsin ${ }^{\mathrm{d}}$ was added to the cell suspension and gently rocked for 10 minutes at room temperature. Subsequently, a solution containing $1.5 \mathrm{ml}$ of trypsin inhibitor and Ribonuclease $\mathrm{A}^{\mathrm{e}}$ was added to the cell suspension and rocked for 10 minutes. Finally, $1.5 \mathrm{ml}$ of an iced propidium iodide solution ${ }^{\mathrm{f}}$ was added under light-protected conditions and rocked. The cell suspension was filtered through a 53 micron nylon mesh into a $12 \times 75 \mathrm{~mm}$ polystyrene tube ${ }^{g}$ protected from light, and cooled on ice until analysis. Samples were analyzed within 3 hours of the addition of the propidium iodide solution. A minimum of 10,000 cells were analyzed by a Becton Dickinson FACScan using CellFIT sofware and doublet discrimination. Histogram analysis of the red fluorescence emitted by the propidium iodide was analyzed manually by setting markers around the $1 \mathrm{~N}, 2 \mathrm{~N}$ and $4 \mathrm{~N}$ peaks and calculating the relative percentages of each ploidy compartment. Known diploid controls were stained in the same procedure as the sample cells to serve as a diploid reference. (For suppliers, see Appendix I.)

\section{Statistical analysis}

Flow cytometry analysis of incisional testicular biopsies was carried out to determine the relative percentage of haploid $(1 \mathrm{~N})$, diploid $(2 \mathrm{~N})$, and tetraploid $(4 \mathrm{~N})$ cells. The percentage of cells in the haploid compartment was compared to the mean value of tubular concentration of spermatids (spermatid/tubule), Sertoli cells (Sertoli cell/ tubule), and the spermatid/Sertoli cell ratio and tubular wall thickness. Normal spermatogenesis, indicated by a haploid predominance, shows a ploidy pattern of $1 \mathrm{~N}>2 \mathrm{~N}>4 \mathrm{~N}$ (Fig 1). A ploidy pattern of $2 \mathrm{~N}>1 \mathrm{~N}>4 \mathrm{~N}$ (Fig 2) reflects abnormal spermatogenesis either by arrested sperm maturation of hypospermatogenesis. Pearson's correlation coefficient was generated from the parameters of quantitative micrometry and DNA flow cytometry analysis. The degree of concordance between each micrometric parameter and the percentage of the germinal cells in the haploid compartment were determined for the entire cohort.

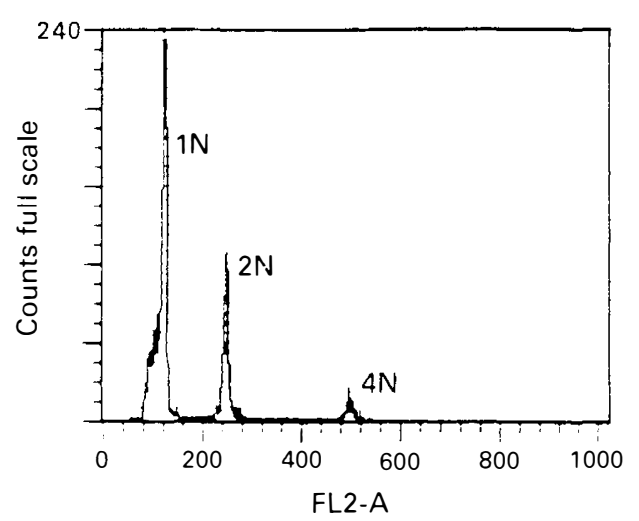

Figure 1 DNA histogram demonstrating normal spermatogenesis with a haploid (1N) predominant cell population.

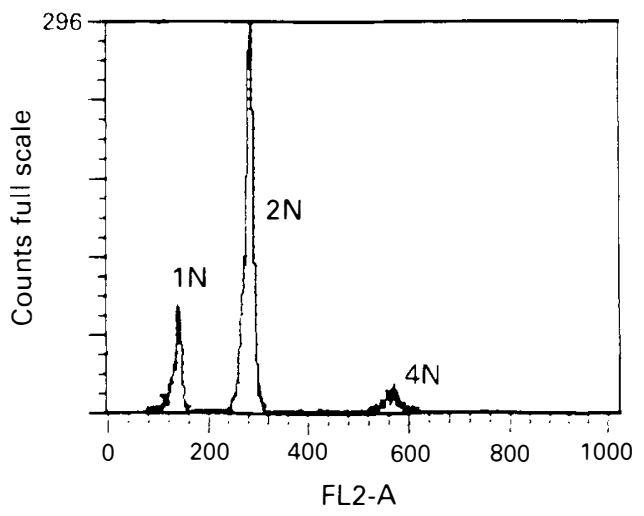

Figure 2 DNA histogram demonstrating spermatogenic arrest with a diploid $(2 \mathrm{~N})$ predominant cell population.

\section{Results}

Analysis of the quantitative micrometric parameters of the 11 SCI patients showed a late spermatid concentration per seminiferous tubule ranging from 4.4 to 25.2 $(x=13.3 \pm 7.3)$. The range of tubular concentration of Sertoli cells was relatively small, from 9.4 to $22.0(x=14.1 \pm 4.1)$. Indexing the spermatid count against the Sertoli cell count within each seminiferous tubule, the spermatid/Sertoli cell ratio provides a useful parameter of spermatogenesis. The mean spermatid/Sertoli cell ratio for this group was $0.86 \pm 0.52$ ranging from 0.09 to 1.59 . Wall thickness of the seminiferous tubule ranged from 0.005 to 0.068 
microns with a mean value of $0.019 \pm 0.02$ microns.

Among the 11 testicular units analyzed, the mean haploid population was calculated at $43 \%$ and ranged from $13 \%$ to $74 \%$. Haploid predominance, indicative of normal spermatogenesis, was noted in six patients and diploid predominance, reflecting spermatogenic insufficiency, was noted in five men. Of these five, three patients demonstrated a severe impairment in spermatogenesis with diploid cell population ranging between $70 \%$ and $74 \%$. Men demonstrating histologically adequate levels of spermatogenesis showed a haploid percentage ranging between $58 \%$ and $74 \%$. Flow cytometry analysis correlated closely with the tubular concentration of spermatids for spinal cord injured men $(r=0.809$, $p<0.002)$ as shown in Figure 3. In addition, a highly significant correlation was noted between the percentage haploidy and the spermatid/Sertoli cell ratio $(r=0.831$, $p<0.0005)$ as shown in Figure 4. The anticipated negative correlation between tubular wall thickness and percent haploid cells was noted as a trend; however, this relationship did not reach statistically significant proportions. There was no observable correlation between either of the quantitative spermatogenic assessment techniques and the degree, duration, or level of spinal cord injury.

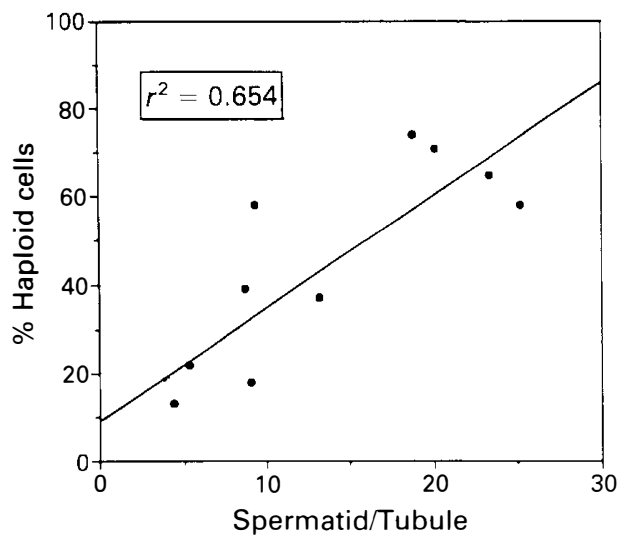

Figure 3 Correlation between flow cytometry analysis of testicular biopsies and the mean number of late spermatids per seminiferous tubule in spinal cord injured men $(p<0.002)$.

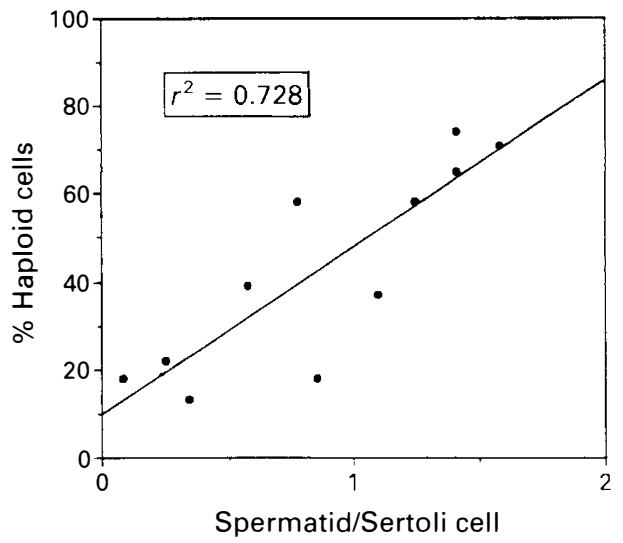

Figure 4 Correlation between flow cytometry analysis of testicular biopsies and the men spermatid/Sertoli cell ratio in spinal cord injured men $(p<0.0005)$.

\section{Discussion}

Neurogenic infertility in spinal cord injured men is of multifactorial etiology attributable to testicular, pretesticular and posttesticular causes. Generalized conditions associated with spinal cord injury which impact significantly on fertility potential are: impotence and ejaculatory dysfunction, ${ }^{8}$ voiding dysfunction, ${ }^{9}$ transient endocrinopathy, ${ }^{10}$ and testicular dysfunction. ${ }^{5}$ Further analysis of semen recovered from spinal cord injured men has shown abnormalities of key constituents of seminal plasma, ${ }^{11}$ leukocytospermia, ${ }^{12}$ poor sperm viability ${ }^{13}$ and poor sperm penetration in cervical mucus and abnormal fertilization that is defined by the hamster egg penetration assay. ${ }^{14}$ As a universal observation in all studies of electrostimulated ejaculates, the finding of asthenospermia (poor sperm mobility) predominates in SCI men with severe asthenospermia (below 20\%) reported in the vast majority of recovered ejaculates. ${ }^{13.15-17}$ While the source of this manifestation of deficient sperm fertilization potential in the setting of SCI has been postulated to arise from denervation of the sperm conduction tract, defective sperm production at the testicular level has been well known for several decades.

Until the recent application of the quanti- 
tative testicular biopsy, the evaluation of spermatogenesis in SCI men has been performed by subjective classification into broad histological categories of normal sperm maturation, arrested maturation, or hypospermatogenesis. These spermatogenic abnormalities were reported in $60-92 \%$ of SCI men studied in various series. In a pioneering study, Bors et al ${ }^{5}$ demonstrated tubular wall thickening and fibrosis, a histological feature indicative of advanced spermatogenic insult, in seven of 34 SCI men. In our previous comparison to fertile able bodied men, SCI was not associated with increased tubular wall thickening. Mean micrometric determination of tubular wall width was similar in both SCI and able bodied cohorts. ${ }^{18}$ The decreased level of objective spermatogenic insult in our recent SCI cohort compared to earlier studies may reflect the intercurrent advances in both urological rehabilitation and general rehabilitative care of SCI patients over the last few decades.

The quantification of spermatogenesis has recently been shown to have significant value in predicting eventual sperm concentration in the electrostimulated ejaculates of spinal cord injured men. ${ }^{19}$ This study noted a significant correlation between objective micrometric parameters of spermatogenesis (quantitative histometry and DNA flow cytometry analysis) and the resultant sperm yield obtained from electroejaculation. The mean tubular concentration of spermatids resulted in a sensitivity and specificity of $100 \%$ and $75 \%$ respectively, as a predictor of sperm yield in recovered semen. Similarly, the mean spermatid/Sertoli cell ratio per seminiferous tubule demonstrated a sensitivity and specificity of $75 \%$ and $87.5 \%$ respectively, in predicting adequate total sperm yield in electrostimulated ejaculates. Thus, the assessment of the germinal epithelium by quantitative methods may ultimately aid in meaningful counseling of spinal cord injured couples with respect to their fertility potential and reproductive choices. Importantly, this information may assist the clinician in the appropriate selection of candidates for invasive semen recovery techniques such as electroejaculation.
Based on the relative constancy of the intratubular Sertoli cell population, Skakkebaek and Heller ${ }^{20}$ examined the value of the spermatid/Sertoli cell ratio as an objective histologic parameter of spermatogenesis in a cohort of known fertile male volunteers. Of the various micrometric parameters, they noted this ratio to have the least intersubject variation. Our present data analyzing the tubular concentrations of Sertoli cells shows a mean value of $14.1 \pm 4.1$ after spinal cord injury and confirms the value of the Sertoli cell compartment as a relatively constant cellular index. While we have previously found a statistically significant correlation in spinal cord injured men between the spermatid/tubule in the sperm concentration in the electrostimulated ejaculate of spinal cord injured men, the relationship between the spermatid/Sertoli cell ratio and electroejaculated sperm has reached even greater proportions of statistical significance. The same relative advantage has been noted in the present study when these micrometric parameters are compared to the percentage of haploid cells on DNA flow cytometry analysis of testicular tissue in spinal cord injured men.

The need for the utilization of objective methodology and standardized techniques in the assessment of spermatogenesis in able bodied infertile men has been widely acknowledged in various clinical studies ${ }^{20}$ To date, this requirement has been fulfilled primarily by the quantitative testicular biopsy with the advantages of objective and reproducible interpretation of data. Clinical application of the quantitative testicular biopsy has been made correlating the tubular concentration of late spermatids with sperm concentration in the ejaculate ${ }^{21}$ and has been extended to the identification of men with partial epididymal obstruction. ${ }^{22}$ In 1973, Skakkebaek and Heller ${ }^{20}$ advanced the parameter of the mean tubular ratio of late spermatids/Sertoli cells, which demonstrated the least intersubject variation of all tubular parameters analyzed histologically. When the tubular ratio of late spermatids/ Sertoli cells was applied to testicular biopsies in a group of spinal cord injured men and fertile controls, we identified this parameter as a powerful discriminator between 
these groups ${ }^{18}$ demonstrating a statistically significant defect in spermatogenesis in spinal cord injured men using these quantitative parameters. However, among other disadvantages, quantitative testicular biopsy by quantitative micrometric methods is a time consuming and labor intensive effort in which some objectivity may be lost by selection of seminiferous tubules for analysis. By contrast, DNA flow cytometry analysis permits a rapid analysis of testicular biopsies (1000 cells/s), an unbiased selection of cell population for analysis, and, importantly, a simple classification and tabulation of each type by its ploidy pattern. These capabilities provide a unique approach to analysis of the seminiferous epithelium which undergoes progressive morphological changes intrinsic to the process of spermatogenesis: mitotic division and proliferation of gonocytes $(2 \mathrm{~N})$, spermatogonia $(2 \mathrm{~N})$, and spermatocytes $(2 \mathrm{~N})$, and reduction division (meiosis) of spermatocytes to produce haploid spermatids $(1 \mathrm{~N})$. Final biochemical and morphological changes transform these late spermatids into testicular spermatozoa in the process of spermiogenesis. Thus, DNA flow cytometry analysis is ideally suited for analyzing testicular cell populations based on their relatively ploidy content: haploid $(1 \mathrm{~N})$, diploid $(2 \mathrm{~N})$, and tetraploid $(4 \mathrm{~N})$. Given the aforementioned broad base of indirect evidence for global insult to the testis and genital tract following spinal cord injury, it is advantageous to refine our selection of patients based on minimally invasive and highly objective methods of assessing spermatogenesis.

DNA flow cytometry analysis has been applied in normal mammalian germinal tissue to investigate age-dependent cellular changes in rodents during the course of normal development from birth to adulthood. ${ }^{23}$ Furthermore, this technique has not resulted in any significant changes in spermatogenesis in rat testes undergoing repeated fine needle aspirations. ${ }^{24}$ Thus, the characterization of normal developmental changes in the growing rodent and the establishment of its reproducibility in longitudinal studies in rodent testis have made DNA flow cytometry analysis a powerful tool in the field of reproductive toxicology with the objective determination of the effects of known gondotoxins. ${ }^{25.26}$

In recent years, the clinical usefulness of DNA flow cytometry in the objective determination of spermatogenesis has been established by correlation of its ploidy patterns with FSH elevation in azoospermic men $^{27}$ and various qualitative histological patterns of spermatogenesis (normal, maturation arrest, hypospermatogenesis, and germinal cell aplasia). ${ }^{6}$ A base of normative data for haploid percentages was established demonstrating a mean value of $46.7 \% \pm 10 \%$ on flow cytometry analysis. ${ }^{27}$ This consistent clinical correlation has identified flow cytometry analysis as useful in the selection of anejaculatory candidates suitable for invasive semen recovery procedures such as electroejaculation. In men with neurogenic infertility, Hellstrom ${ }^{28}$ observed normal ploidy content in the biopsies of $80 \%$ of men with favourable semen parameters and abnormal ploidy content in $80 \%$ of men with abnormal semen parameters in electroejaculates.

The present study tests the applicability of DNA flow cytometry analysis as an alternative to quantitative micrometry, the current standard of spermatogenic assessment in the objective evaluation of human spermatogenesis following SCI. By direct comparison to the micrometric parameters which comprise the quantitative testicular biopsy, we have demonstrated a statistically significant correlation between the percentage of haploid cells on DNA flow cytometry analysis and the important quantitative parameters of the quantitative testicular biopsy: the mean tubular concentration of late spermatids, and the spermatid/Sertoli cell ratio per seminiferous tubule.

\section{Conclusion}

In the able bodied infertile male population, DNA flow cytometry analysis has demonstrated distinct clinical advantages over the incisional testicular biopsy because it can be easily accomplished by needle aspiration of the testis. Therefore, this office-based procedure is less invasive, more cost effective and safer than conventional testicular biopsy. Rapid, reproducible and objective 
interpretation of the data is accomplished by flow cytometry in an operator-independent fashion, thereby minimizing any subjective factors intrinsic to the conventional quantitative testicular biopsy. With the widespread use of flow cytometry in major medical centers where reproductive rehabilitation programs are currently based, this technique can easily be applied to our current efforts in this field.

\section{Appendix I}

\section{Suppliers}

"Hank's Balanced Salt Solution, \#310-4185 AJ. Gibco. 3175 Staley Road, Grand Island, NY
14072.

${ }^{\mathrm{b} C y c l e}$ TEST Kit \#95-2000, Becton Dickinson Immunocytometry Systems, 2350 Qume Drive, San Jose, CA 95131-1807.

'Polypropylene freezing vials, \#26702, Corning Inc. Corning, NY 14831.

${ }^{\mathrm{d} C y c l e}$ TEST Solution A, Becton Dickinson Immunocytometry Systems, 2350 Qume Drive, San Jose. CA 95131-1807.

'Cycle TEST Solution B, Becton Dickinson Immunocytometry System, 2350 Qume Drive, San Jose, CA 95131-1807.

${ }^{\mathrm{t}}$ Cycle TEST Solution C, Becton Dickinson Immunocytometry System, 2350 Qume Drive, San Jose, CA 95131-1807.

gPolystyrene tube, Falcon 2052. Becton Dickinson and Co, 2 Bridgewater Lane. Lincoln Park, NJ 07035.

\section{References}

1 National Spinal Cord Injury Statistical Center (1988) Spinal Cord Injury. University of Alabama at Birmingham. AL.

2 Cole TM, Edgerton VR (1990) Report of the task force on medical rehabilitation research. Hunt Valley, Maryland.

3 Seager SWJ. Halstead LS. Ohl DA. Bennett CJ. Lipshultz LI. Belker AM et al (1990) Electrocjaculation and its utilization for fertility evaluation in 266 spinal cord injured men. American Spinal Injury Association. Orlando, FL. May 4. 1990.

4 Ohl DA, Randolph JF, Bennett CJ, Menge AC. Ayers WT (1990) Combined electroejaculation and in-vitro fertilization for sperm function assessment and treatment of anejaculatory infertility. J Urol 143: $345 \mathrm{~A}$.

5 Bors E. Engle ET, Rosenquist RC. Hollinger VH (1950) Fertility in paraplegic males: a preliminary report of endocrine studies. J Clin Endocrinol 10: $381-98$.

6 Kaufman DG and Nagler HM (1987) Aspiration flow cytometry of the testis in evaluation of spermatogenesis in infertile males. Fertil Steril 48: 287-91.

7 Clermont Y (1963) The cycle of the seminiferous epithelium in man. Am J Anat 112: 35-47.

8 Griffiths ER, Tomko MA. Timms RJ (1973) Sexual function in spinal cord injured patients: a review. Arch Phy's Med Rehabil 54: 539-543.

9 Ohl DA. Bennett CJ, McCabe J. Menge AC. McGuire EJ (1989) Predictors of success in electroejaculation of spinal cord injured men. J Urol 142: 1438-6.

10 Claus-Walker J. Scurry M, Carter RE, Campos RJ (1977) Steady state hormonal secretion in traumatic quadriplegia. J Clin Endocrinol Metab 44: 530-5.

11 Hirsch IH, Jeyendran RS. Sedor J, Rosecrans RR. Staas WE (1991) Biochemical analysis of electroejaculates in spinal cord injured men: comparison to normal ejaculates. J Urol 145: 73-6.

12 Sedor J, Hirsch IH. Shupp-Byrne D, Levinson M (1992) Immunohistologic characterization of leukocytes in electroejaculates. J Androl 13: 52P.

13 Hirsch IH, Sedor J, Jeyendran RS, Staas WE (1992) The relative distribution of viable sperm in the antegrade and retrograde portions of ejaculates obtained after electrostimulation. Fert Steril 57: 399-401.

14 Denil J, Ohl DA, Menge AC. Keller LM, McCabe M (1992) Functional characteristics of sperm obtained by electroejaculation. J Urol 147: 69-72.

15 Bennett CJ. Seager SW, Vasher EA. McGuire EJ (1988) Sexual dysfunction and electroejaculation in men with spinal cord injury: review. J Urol 139: 453-7.

16 Halstead LS, VerVoort SM, Seager SWJ (1987) Rectal probe electrostimulation in the treatment of anejaculatory spinal cord injured men. Paraplegia 25: 120-9.

17 Perkash I, Martin DE, Warner H, Blank MS, Collins DC (1986) Reproductive biology and serum hormone evaluation. J Urol 134: 284-7.

18 Hirsch IH, McCue P, Allen J, Lee J, Staas WE (1991) Quantitative testicular biopsy in spinal cord injured men: comparison to fertile controls. J Urol 146: 337-41.

19 Hirsch IH, Kulp D, McCue P, Flanigan M, Sedor J, Stevenson A et al (in press) The value of quantitative testicular biopsy and DNA flow cytometry in predicting sperm recovery from electrostimulated ejaculates. $J$ Urol.

20 Skakkebaek NE, Heller CG (1973) Quantification of human seminiferous epithelium. J Reprod Fertil 32: $379-89$. 
21 Zuckerman Z, Rodriquez-Rigau LJ, Weiss DB (1978) Quantitative analysis of the seminiferous epithelium in human testicular biopsies, and the relation of spermatogenesis to sperm density. Fertil Steril 30: 448-55.

22 Silber SJ, Rodriguez-Rigau LJ (1981) Quantitative analysis of testicular biopsy: determination of partial obstruction and prediction of sperm count after surgery for obstruction. Fertil Steril 36: 379-85.

23 Clausen OP, Purvis K. Hansson V (1977) Application micro-flow flourometry to studies of meiosis in the male rat. Biol Reprod 17: 555-60.

24 Skoog SJ, Evans CP, Hayward IJ, Griffin JL. Hitchcock CL (1991) Flow cytometry of fine needle aspiration of the Sprague-Dawley rat testis: defining normal maturation and the effect of multiple biopsies. J Urol 146: 620-3.

25 Evenson DP, Higgin PJ, Gruenegerg D. Ballachey BE (1985) Flow cytometric analysis of mouse spermatogenic function following exposure to ethylnitrosuourea. Cytometry 6: 238-53.

26 Hacker-Klom U (1985) Long-term effects of ionizing radiation on mouse spermatogenesis. Acta Radiol Oncol 4: 363-7.

27 Abyholm T and Clausen OPF (1981) Clinical evaluation of DNA flow cytometry of fine needle aspirates from testes of infertile men. Int $J$ Androl 4: 505-14.

28 Hellstrom WJG, Stone AR, Deitch AD, deVere White RW (1989) The clinical application of aspiration deoxyribonucleic acid acid flow cytometry to neurologically impaired men entering an electroejaculation program. J Urol 142: 309-12. 